\title{
Mycobacterium simiae Infection in a Patient with Acquired Immunodeficiency Syndrome
}

\author{
J.L.M.Sampaio, N. Artiles, R.M.G.Pereira, \\ J.R.Souza and J.P.G.Leite
}

\author{
Lâmina Laboratory, Rio de Janeiro, RJ; Ferreira \\ Machado Hospital, Campos, RJ; FIOCRUZ, Rio de \\ Janeiro, RJ; Fleury - Centre of Diagnostic Medicine, \\ São Paulo, SP, Brazil
}

\begin{abstract}
Mycobacterium simiae is usually an environmental contaminant rarely associated with human disease. We report a fatal case of M.simiae infection in a 37 year old, HIV positive, male from whom the organism was isolated from blood culture. The identification of M.simiae was performed using DNA amplification followed by analysis on 3\% agarose gel of the amplicon fragments after digestion by restriction endonucleases. The precise identification of mycobacterial isolates to the species level is important, with both epidemiological and therapeutic implications.

Key Words: Mycobacterium simiae, AIDS, RFLP.
\end{abstract}

Since most laboratories in Brazil do not identify Mycobacterium other than tuberculosis (MOTT) to the species level, and considering that the most frequent MOTT is Mycobacterium avium-intracellulare complex (MAI complex), patients are treated empirically for this group of agents when there is a laboratory report of MOTT.

The number of different organisms included in the category MOTT, is growing, and commercially available products to detect yheir diversity do not fill the Mycobacteria laboratory need for species identification.

The only description of M.simiae isolated in Brazil was reported in 1995, in Araraquara, SP [1]. It was isolated from sputum of an HIV positive patient and there was no clinical history and no report of outcome. To our knowledge, this is the first report of M.simiae isolated from blood in an AIDS patient in Brazil. The

Received on 15 August 2001; revised 16 September 2001.

Address for correspondence: Dr. Jorge Luiz Mello Sampaio. Fleury Centro de Medicina Diagnóstica. Av. Gal. Waldomiro de Lima, 508, São Paulo/SP, Brazil, Zip Code: 04344-070. Phone: (55 11) 5014-7730. Fax:(55 11)5014-7601 .

E-mail: jorge.sampaio@fleury.com.br

The Brazilian Journal of Infectious Diseases 2001;5(6):352-355. (C) 2001 by The Brazilian Journal of Infectious Diseases and Contexto Publishing. All rights reserved. 1413-8670 intent of this report is to show the need to identify Mycobacterium to the species level in all clinical isolates. Such identification will have both epidemiological and treatment implications.

\section{Case Report}

A 37-year-old man was found to be HIV seropositive 5 years before the diagnosis of M.simiae infection. At the first hospital admission, he presented weight loss, diarrhea, and oral candidiasis. At that time, Zidovudine ${ }^{\mathrm{TM}}$ and Didanosin ${ }^{\mathrm{TM}}$ were initiated. His general condition improved but, due to erratic use of medication, he was admitted to the Infectious Disease Service at Ferreira Machado Hospital, Campos, Rio de Janeiro State, Brazil, in August, 1998, presenting persistent fever, diarrhea, progressive weight loss, and gray skin. At hospital admission, laboratory tests showed: hematrocrit $28 \%$, hemoglobin $9.5 \mathrm{~g} / 100 \mathrm{~mL}$, total leukocytes count $2,400 / \mathrm{mm}^{3}$ and $\mathrm{CD}^{+}$count $146 / \mathrm{mm}^{3}$. A bone marrow aspirate was done but the only alteration detected was hypoplasia. Cotrimoxazol ${ }^{\mathrm{TM}}$ was prescribed, and the patient's general condition improved. He was discharged using Zidovudine ${ }^{\mathrm{TM}}$, 
Lamivudine $^{\mathrm{TM}}$, Indinavir ${ }^{\mathrm{TM}}$, Ketoconazol ${ }^{\mathrm{TM}}$ and Cotrimoxazol $^{\mathrm{TM}}$. During his outpatient follow-up special blood cultures for Mycobacterium sp. and fungi were collected.

The patient was lost to medical care for the next 2 months, but then his general condition worsened. At that time he had significant weight loss, persistent fever, hepatosplenomegaly, diarrhea and a positive blood culture for MOTT. He was admitted to the Infectious Disease Service at Ferreira Machado Hospital on November 18, 1998. Laboratory tests showed: hematocrit $21 \%$, hemoglobin $6.8 \mathrm{~g} / 100 \mathrm{~mL}$, total leukocytes count $1,900 /$ $\mathrm{mm}^{3}$, and platelet count $78,000 / \mathrm{mm}^{3}$. Amikacin ${ }^{\mathrm{TM}}$, Ethambutol $^{\mathrm{TM}}$, Chlaritromycin ${ }^{\mathrm{TM}}$ and Ciprofloxacin ${ }^{\mathrm{TM}}$ were introduced. After 17 days of hospital stay, he was sent home without fever or diarrhea.

On December 22, 1998, he returned with a recurrence of the signs and symptoms similar to those at last hospital admission. He mentioned that he had used his medication erratically. With regular use of Ciprofloxacin $^{\mathrm{TM}}$ (1g/daily), Ethambutol ${ }^{\mathrm{TM}}$ (1.2g/daily) and Chlarithromycin ${ }^{\mathrm{TM}}(1.0 \mathrm{~g} /$ daily $)$, his clinical condition improved and he was sent home. One month later his general condition worsened and soon after rehospitalization, he died.

\section{Isolation and identification procedures}

Five $\mathrm{ml}$ of blood were cultivated on each 13A radiometric media and analyzed with Bactec $460 \mathrm{~TB}$ (Becton-Dickinson). The vial was incubated at $37^{\circ} \mathrm{C}$ and read 3 times a week during the first 2 weeks. The vial was positive at the end of the second week. The microscopic examination showed numerous acid fast staining bacteria. A sample of liquid media was planted on Lowenstein-Jensen medium for additional tests. After DNA extraction, a polymerase chain reaction (PCR) was carried out using the set of primers and the protocol described previously by Telenti, et al., [2]. The expected amplicons of 439 base pairs (bp) were visualized on $2 \%$ agarose (Life Technologies) gel stained with ethidium bromide $(0.05 \mu \mathrm{g} / \mathrm{mL})$. The amplicons were digested with BstEII and HaeIII restriction endonucleases, following the manufacturer's recommendations (Life Technologies) [2]. The products of BstEII and HaeIII cleavage were analyzed on 3\% Metaphor $^{\mathrm{TM}}$ (FMC Bioproducts) agarose gel electrophoresis. M. simiae ATCC 29275 and $M$. tuberculosis ATCC 27294 prototype strains were used as control (Figure 1). The strain isolated from the patient showed exactly the same polymorphism restriction analysis (PRA) pattern of M.simiae ATCC 29275. The isolate was confirmed as $M$. simiae at the Health Center at Tyler, University of Texas, USA.

\section{Discussion}

M.simiae is a photochromogenic environmental acid-fast bacillus that has rarely been associated with human disease. However, pulmonary infections due to this organism have been reported in monkey handlers and in people who have had contact with these primates. It was originally isolated in 1965, from monkeys imported from India to Hungary [3]. Since then, M. simiae has been isolated from tap water and soil samples in the Middle East, Central Africa, USA, and Australia [4,5]. In Germany, it has been isolated from water systems from dental units [6].M. simiae is a common isolate from clinical specimens in Israel [7] and has been isolated from the water supply in Gaza [8]. In Europe, M simiae has been isolated from $28 \%$ of stool specimens from 50 volunteers [9]. Reports of M.simiae infection in AIDS patients have been previously described $[9,10]$. The isolate was resistant to all common antituberculous chemotherapeutic agents, as were M.simiae from other case reports elsewhere.

Until now, we were not able to find any report on isolation of $M$. simiae from Brazil. In Brazil, the vast majority of mycobacterial isolates are reported as $M$. tuberculosis complex, or MOTT, when a culture is performed. In fact, most primary care public health institutions in Brazil do not perform cultures on patients with clinical diagnosis of tuberculosis. Diagnosis is made only by X-ray and acid fast smear tests from sputum.

Leite, et al. studied 78 mycobacterial isolates from patients with suspected pulmonary tuberculosis during 
Figure 1. Polymorphism restriction analysis of amplicons of $439 \mathrm{bp}$ digested with BstEII and HaeIII restiction endonucleases.

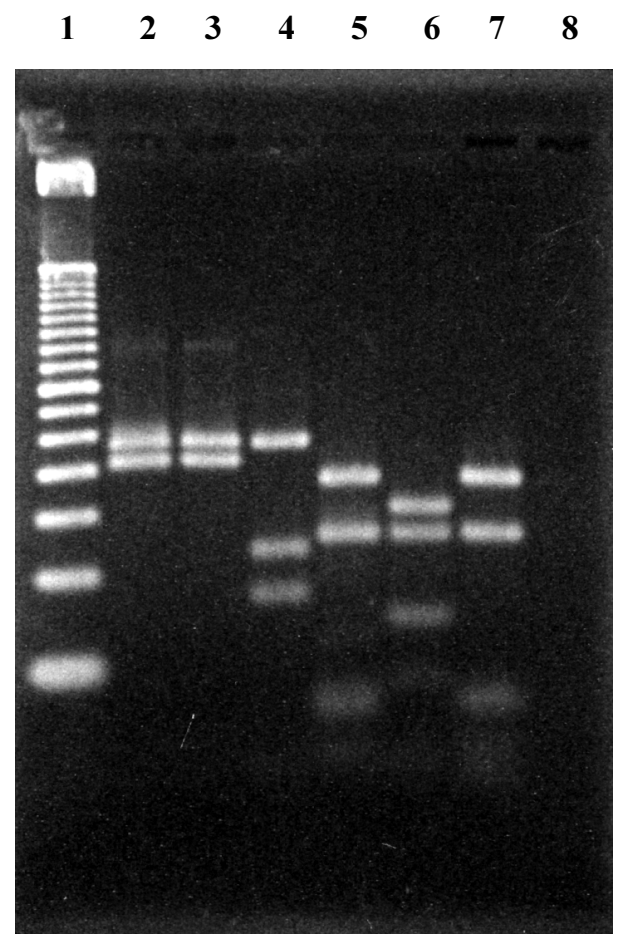

Lane 1: MW 50bp DNA ladder (Life Technologies); Lanes 2-4: BstEII restriction endonuclease digestion of amplicons from $M$. simiae (patient), M. simiae ATCC 29275, and M.tuberculosis ATCC 27294; Lanes 5-7: HaeIII restriction endonuclease digestion of amplicons of M. simiae (patient), M. tuberculosis ATCC 27294, and M. simiae ATCC 29275.

the year of 1993, in Araraquara city in São Paulo state. The method used was thin layer chromatography of mycolic acids and some biochemical tests. Among 78 isolates, 69 were M.tuberculosis, 5 were M.aviumintracelullare, 2 were M.fortuitum, 1 was M.chelonae and 1 was M.simiae. M.tuberculosis was the most prevalent among both HIV negative $(88.4 \%)$ and HIV positive $(53.3 \%)$ groups. There was only 1 M.simiae isolate and it was from HIV positive patient.

It is not always easy to differentiate M. simiae from M.scrofulaceum based on traditional tests like pigment and niacin production, so chromatography or molecular methods must be used. In our laboratory we are using molecular methods to identify all isolates of Mycobacterium spp. From January, 1999, to December, 2000, from a total of 256 mycobacterial isolates, $162(63.3 \%)$ belong to the M.tuberculosis complex, and $94(36.7 \%)$ were non-Tuberculous Mycobacteria (NTM). Among NTM, 2 were identified as M.simiae, 1 isolated from blood, and 1 from bronchoalveolar lavage, representing $2.13 \%$ of all NTM isolates. Therefore, we believe that M.simiae is a present, but under diagnosed, infection.

\section{References}

1. Leite C.Q.F., Viana B.H.J., Leite S.R.A., Juarez E. Incidence of Mycobacterium tuberculosis and other mycobacteria on pulmonary infections in AraraquaraSP, 1993. Rev Microbiol 1995;26:101-5.

2. Telenti A., Marchesi F., Balz M., et al. Rapid identification of Mycobacteria to the species level by polymerase chain reaction and restriction enzyme analysis. J Clin Microbiol 1993;31:175-8. 
3. Karassova V., Weissfeiler J., Krasznay E. Occurrence of atypical mycobacteria in Macacus rhesus. Acta Microbiol Ac Sci Hung 1965; 12:275-82.

4. Lavy A., Rusu R., Shaheen S. Mycobacterium simiaeintracellulare in clinical specimens: etiological factor or contaminant. Isr J Med Sci 1990;26:374-8.

5. Portaels F., Walsh G.P., De Ridder K., et al. Cultivable mycobacteria isolated from 32 newly captured armadillos (Dasypus novemcinctus) from Louisiana. Int J Lepr 1987;55:788.

6. Schulze-Röbbecke R., Feldmann C., Fischeder R., et al. Dental units: an environmental study of sources of potentially pathogenic mycobacteria. Tubercle Lung Dis 1995;76:318-23.

7. Lavy A., Yoshpe-Purer Y. Isolation of Mycobacterium simiae from clinical specimens in Israel. Tubercule 1982;63:279-85.

8. Lavy A., Rusu R., Shaheen S. Mycobacterium aviumintracellulare in clinical specimens: etiological factor or contaminant? Isr J Med Sci 1990;26:374-8.

9. Vandercam B., Gala J., Vandeweghe B., et al. Mycobacterium simiae disseminated infection in a patient with acquired immunodeficiency syndrome. Infection 1996;24:49-51.

10. Huminer D., Dux S., Samra Z., et al. Mycobacterium simiae Infection in Israeli Patients with AIDS. Clin Infect Dis 1993; 17:508-9. 